This item was submitted to Loughborough's Research Repository by the author.

Items in Figshare are protected by copyright, with all rights reserved, unless otherwise indicated.

\title{
An experimental study of unsteady vehicle aerodynamics
}

PLEASE CITE THE PUBLISHED VERSION

PUBLISHER

(c) IMechE / Professional Engineering Publishing

VERSION

VoR (Version of Record)

LICENCE

CC BY-NC-ND 4.0

REPOSITORY RECORD

Passmore, Martin A., S. Richardson, and A. Imam. 2009. "An Experimental Study of Unsteady Vehicle Aerodynamics". figshare. https://hdl.handle.net/2134/4614. 
This item was submitted to Loughborough's Institutional Repository (https://dspace.lboro.ac.uk/) by the author and is made available under the following Creative Commons Licence conditions.

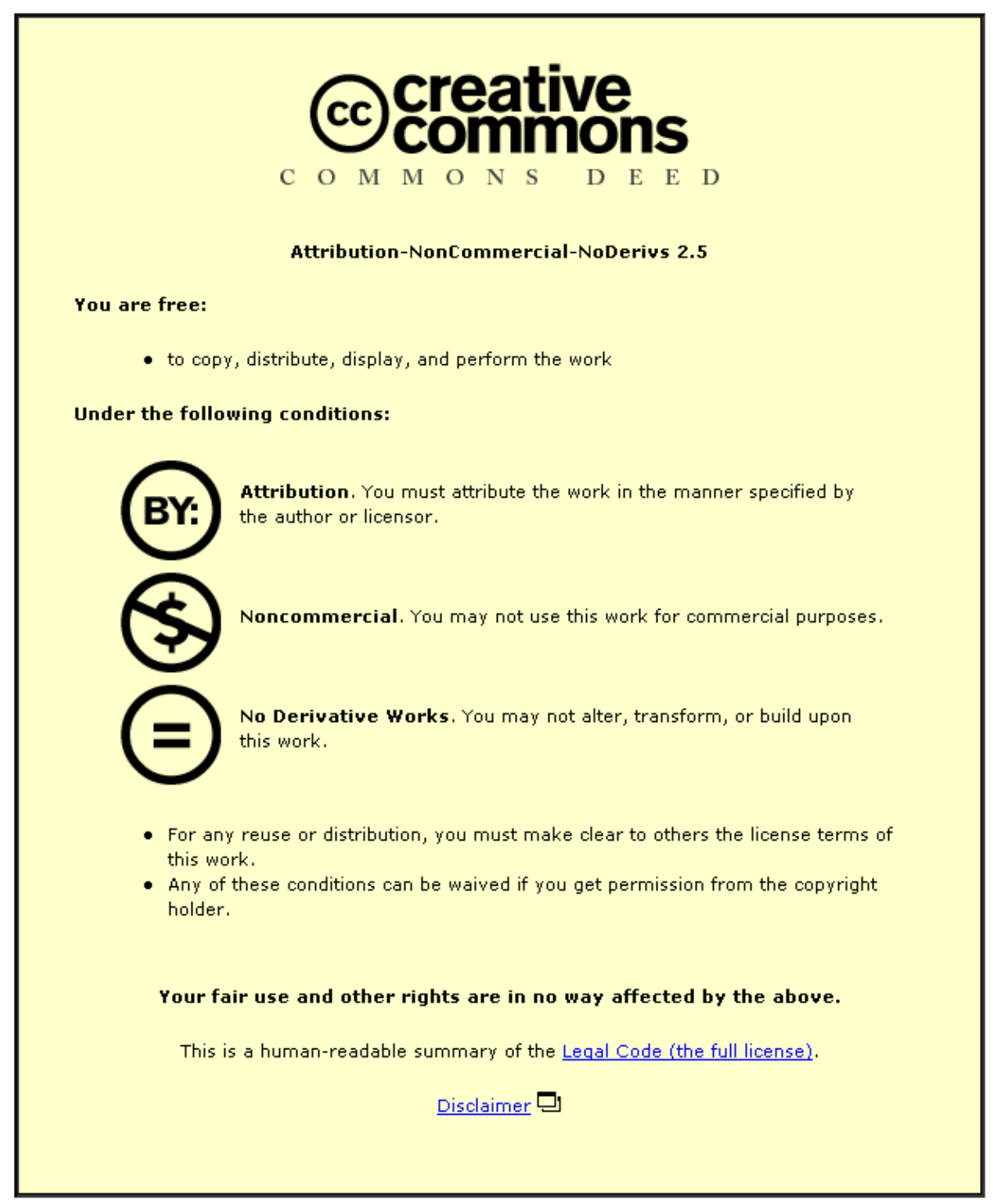

For the full text of this licence, please go to: http://creativecommons.org/licenses/by-nc-nd/2.5/ 


\title{
An experimental study of unsteady vehicle aerodynamics
}

\author{
M A Passmore*, S Richardson and A Imam
}

Department of Aeronautical and Automotive Engineering, Loughborough University, Leicestershire, UK

\begin{abstract}
The transient response of a vehicle to a wind disturbance is of importance to car drivers since low level inputs can result in poor vehicle refinement, and extreme effects can result in path deviation. This paper investigates the use of an oscillating aerofoil gust generator to simulate the transient aerodynamic effects produced on a car-type bluff body during a simplified sinusoidal side gust interaction. A simplified bluff body was exposed to a range of sinusoidal cross-wind excitations corresponding to a reduced frequency of between 0.09 and 0.71 based on the model length. Unsteady measurements of surface pressure are processed to determine the side force and yaw moment and the aerodynamic magnification $\left(\chi_{\mathrm{a}}\right)$ is calculated by comparing the transient response with a quasisteady prediction. The transient yaw moment response is shown to exceed the quasi-steady by as much as 30 per cent. The transient side force is generally significantly less than the quasi-steady value except at the lowest frequency tested. The change in response is attributed to changes in the strength of the front and rear pillar vortices and to changes in phase relative to the quasi-steady response.
\end{abstract}

Keywords: vehicle cross-wind stability, cross-wind sensitivity, unsteady aerodynamics, admittance, oscillating aerofoil, side force, yaw moment

\section{NOTATION}

$A_{\mathrm{g}} \quad$ non-dimensional gust amplitude

$A_{0} \quad$ maximum aerofoil angle (rad)

$C_{\mathrm{M} z} \quad$ coefficient of yaw moment

$C_{p} \quad$ coefficient of pressure

$C_{y} \quad$ coefficient of side force

$f \quad$ frequency $(\mathrm{Hz})$

$k_{\mathrm{m}} \quad$ model reduced frequency

$l$ model characteristic length $(\mathrm{m})$

$n \quad$ tapping number

$t \quad$ time (s)

$u \quad$ longitudinal oscillation flow component $(\mathrm{m} / \mathrm{s})$

$U$ tunnel freestream speed $(\mathrm{m} / \mathrm{s})$

$v \quad$ cross-component of velocity $(\mathrm{m} / \mathrm{s})$

$\beta \quad$ yaw angle (rad)

$\chi_{\mathrm{a}} \quad$ aerodynamic magnification factor

$\psi \quad$ phase angle (rad)

\section{INTRODUCTION}

The unsteady or transient response of a vehicle to a wind disturbance is of importance to car drivers on two levels. Moderate effects can result in poor vehicle refinement and extreme effects can result in path deviation, generating concern about vehicle safety. Road vehicles encounter unsteady cross-wind disturbances in a number of situations [1]; the cross-wind profile measured from a moving vehicle on a windy day will show characteristic patterns corresponding to the topography of roadside features and the presence of other vehicles. A large fetch of rough terrain, for example, introduces large-scale turbulence and has the effect of creating a random gust profile. Bridges and overtaking trucks develop sudden step changes while cuttings produce more gradual changes to the cross-wind. The conditions met by vehicles under normal rather than extreme driving conditions are summarized by Watkins and Saunders [2]. Spectral analysis indicated the peak energy at approximately $1 \mathrm{~Hz}$ but showed that it could vary between 0.25 and $2.5 \mathrm{~Hz}$. At motorway speeds this corresponds to a reduced frequency of between 0.09 and 0.9 . Here, for consistency with previously published work [3], reduced frequency is defined as

$$
k_{\mathrm{m}}=\frac{\pi f l}{U}
$$

The MS was received on 1 November 2000
revision for publication on 7 February 2001.

* Corresponding author: Department of Aeronautical and Automotive Engineering, Loughborough University, Loughborough, Leicestershire LE11 $3 T U, U K$. 
where

$f(\mathrm{~Hz})=$ frequency

$l=$ characteristic length (vehicle length in metres)

$U(\mathrm{~m} / \mathrm{s})=$ freestream airspeed

The development of streamlined, or low drag, car bodies to satisfy the demand for better fuel economy has tended to increase the sensitivity of vehicles to cross-wind disturbances. This additional sensitivity arises because the drag reduction techniques have generally involved rounding of the front-end profiles and the region around the rear pillar. In the presence of crosswinds this can give rise to large differences in front and rear side forces and hence generate yaw moments that tend to destabilize the vehicle. Under unsteady conditions the added body curvature can also lead to uncertain and variable separation resulting in unsteady aerodynamic loads. Despite the relatively high mass and the sharper edge radii normally seen, the now ubiquitous four by four or utility vehicles are not exempt from the cross-wind stability problem. The coupling of the roll and yaw motions, through the suspension non-linearity, can cause path deviations when a large aerodynamic roll moment is generated. There is clearly a need to improve the understanding of the unsteady case and for the development of techniques to measure and quantify a vehicle's susceptibility to cross-wind inputs at an early stage in the development.

At full scale two main sources of information are commonly available. Steady state tunnel tests conducted at yaw generate useful initial data but there is insufficient evidence at present to correlate this adequately with performance in a transient situation. Alternatively, the vehicle is driven through an artificial gust generated by a collection of fans or jets. Lateral acceleration, yaw rate, steering and overall deviation are monitored and the vehicle response is characterized. The method is effective for the purposes of evaluating an existing design, but cannot be performed until a stage in the vehicle development process is reached when it is too late to effect appropriate changes.

At model scale there are a number of techniques available. The best known [4] simulates the cross-wind interaction by propelling the model along a track across the working section of a wind tunnel. This technique also makes it possible to simulate the ground boundary layer, although in many cases this is not implemented because of the additional difficulty of interpreting the results. Macklin et al. [4] conclude that in general the static loads are a conservative estimate of the dynamic case at yaw angles below approximately $15^{\circ}$. However, the figures presented suggest that for some test configurations the peak yaw moments are as much as 30 per cent higher than the static values at quite low yaw angles. Above $15^{\circ}$ of yaw there are considerable overshoots in yaw moment and above $30^{\circ}$ there are overshoots in the side force. The difficulty with the method is that the model scale must be small if appropriate gust lengths are to be investigated, thereby limiting the test Reynolds number. Further, since the balance is connected to the moving model the induced noise and vibration create a major interference and design problem. An alternative approach [5] oscillated the model about its vertical axis: the magnitude of the results was similar to that for static data but a lag was introduced that was a function of frequency of oscillation. This method also suffered from poor signal-to-noise ratios.

Using a static model the potential for improved signalto-noise ratio is enhanced. Ryan and Dominy [6] used a cross-wind gust generated within a wind tunnel working section by the application of an additional nozzle to create a cross-flow. A shutter spool exposes the model to a near square wave gust profile. On integrating the results from surface pressure tappings it is shown that the transient side force coefficient exceeds the corresponding steady state value by between 10 and 20 per cent. However, there is no corresponding overshoot in the yaw moment.

Bearman and Mullarkey [3] generated sinusoidal gusts using oscillating aerofoil sections positioned upstream of a stationary model. A range of reduced frequencies was achieved by varying the aerofoil oscillation frequency and the tunnel speed. A transverse component of the gust is convected downstream to the test model with the freestream. The results are presented in the form of an aerodynamic admittance function [7]: the ratio of the dynamic power to the power predicted from quasi-steady data. The experiments produced the unexpected result that at low frequencies the admittance did not tend to unity for either side force or yaw moment. At higher frequencies the side force admittance was always less than unity, but the yaw moment admittance exceeds unity for non-dimensional frequencies above approximately 1.3. Bearman and Mullarkey conclude from the work that in general it is sufficient to make conventional steady state measurements as they provide a conservative estimate of the dynamic loads.

The advantage of the sinusoidal gust generator is that it allows in-depth study of transient phenomena with a repeatable, single-frequency, cross-wind perturbation. In addition, the ability to generate plots of aerodynamic response, over a range of appropriate frequencies, may provide a means of comparing the cross-wind sensitivities of different vehicle configurations early in the design process. These characteristics encouraged the development of such a facility for the work reported here. Initially the facility was used to measure side force and yaw moment admittance using an internal balance. However, considerable problems of signal-to-noise ratio were encountered, particularly at low frequencies, and the work has not been reported. This paper reports the results of an alternative method using surface pressure tappings to allow the calculation of the pressure component of side force and the associated yawing moment. 
Using a simplification in the analysis the results are reported as aerodynamic magnification factors [7], the ratio of transient to predicted quasi-steady force or moment, rather than aerodynamic admittance. In this form the results are more readily interpreted by aerodynamicists and handling specialists. The application of surface tappings also facilitates analysis of the steady and transient pressure fields and consideration of the phase changes which occur along the model during a gust.

\section{EXPERIMENTAL SET-UP}

\subsection{Aerofoil gust generator}

The gust generator employed in this work is based extensively on that described by Mullarkey [8], although it is somewhat larger, allowing a larger model scale, and can be operated across the relevant frequency range at a single Reynolds number. It is installed in the upstream part of the closed working section of a closed-circuit wind tunnel. The test model is located downstream, between the aerofoils, in an irrotational region away from the wake of the aerofoils. The tunnel working section is $1.6 \mathrm{~m}$ wide by $1 \mathrm{~m}$ high and $4.7 \mathrm{~m}$ in length. Figure 1 is a pictorial representation of the experimental set-up.

The primary requirement of the design is to generate a large value of $A_{\mathrm{g}}$, the non-dimensional side gust component, which is important in producing sizeable gusts. This is dependent on the span, chord, the distance $\left(y_{0}\right)$ between the aerofoils and the operating frequency.

The span of the aerofoils was fixed to the height of the wind tunnel, to limit end effects, and $y_{0}$ was calcu- lated such that a 1/6 scale vehicle model would be contained in the core flow. The $0.3 \mathrm{~m}$ chord NACA0015 aerofoils are oscillated in phase about a spanwise pivot located at quarter chord. The gust generator is driven by a $15 \mathrm{~kW}$ d.c. motor and controller with encoder feedback. It can be controlled from close to zero speed up to an oscillation frequency of $18 \mathrm{~Hz}$ (equivalent to a wavelength of approximately 2 car lengths at a tunnel operating speed of $22 \mathrm{~m} / \mathrm{s}$ ). This upper frequency relates to a Strouhal number of 0.23 above which vortex shedding is very weak. Drive to the aerofoils is via a grooved cam providing sinusoidal motion. The peak combined inertial and aerodynamic load on each aerofoil is approximately $1300 \mathrm{~N}$ at the maximum frequency.

Calibration of the gust generator was achieved using a constant temperature anemometer employing a twocomponent split film sensor. Initial calibration of the probe was obtained by yawing it in the tunnel. Data were then obtained with the gust generator operating over a range of frequencies and the sensor was positioned to coincide with the position of the front of the test model. This was processed to produce $v$ and $U+u$ components from which the non-dimensional gust amplitude $\left(A_{\mathrm{g}}\right)$ is derived:

$$
A_{\mathrm{g}}=\frac{v}{(u+U) A_{0}}
$$

The calibration data shown in Fig. 2 are presented as a function of reduced frequency based on the model length to assist later interpretation.

\subsection{Model and instrumentation description}

The model used is a replica of the Davis model [9] (Fig. 3) and corresponds to approximately a one-sixth
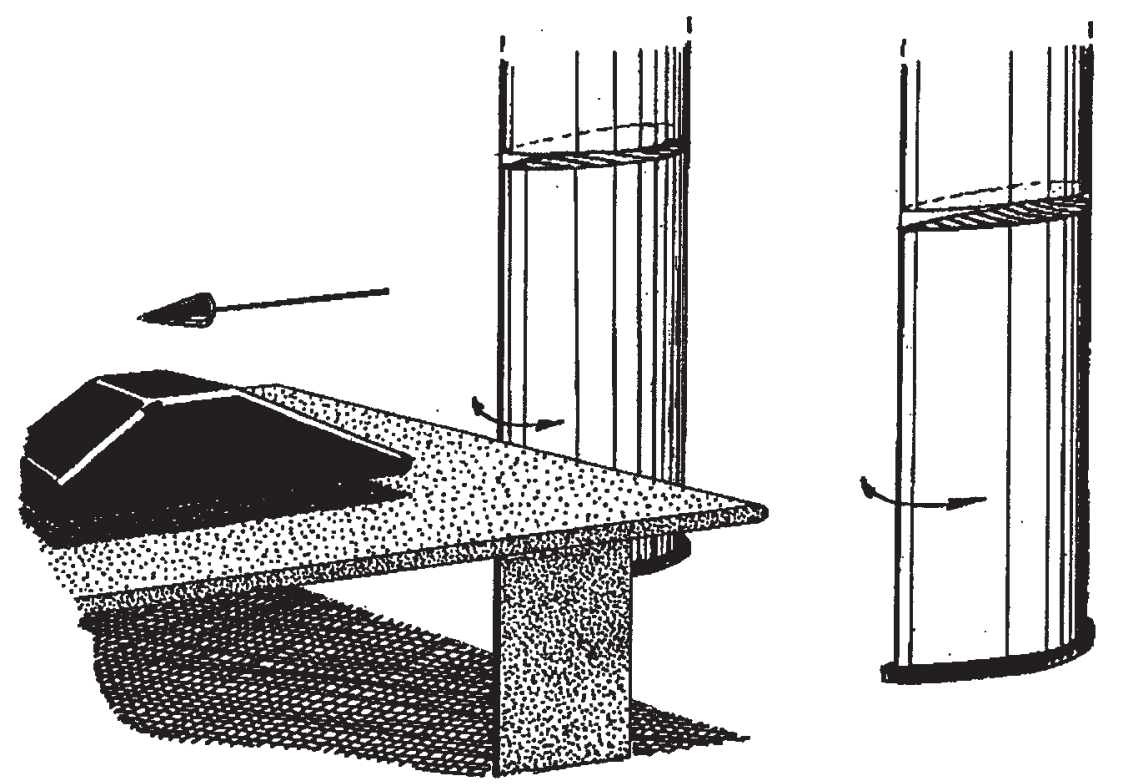

Fig. 1 The experimental set-up 


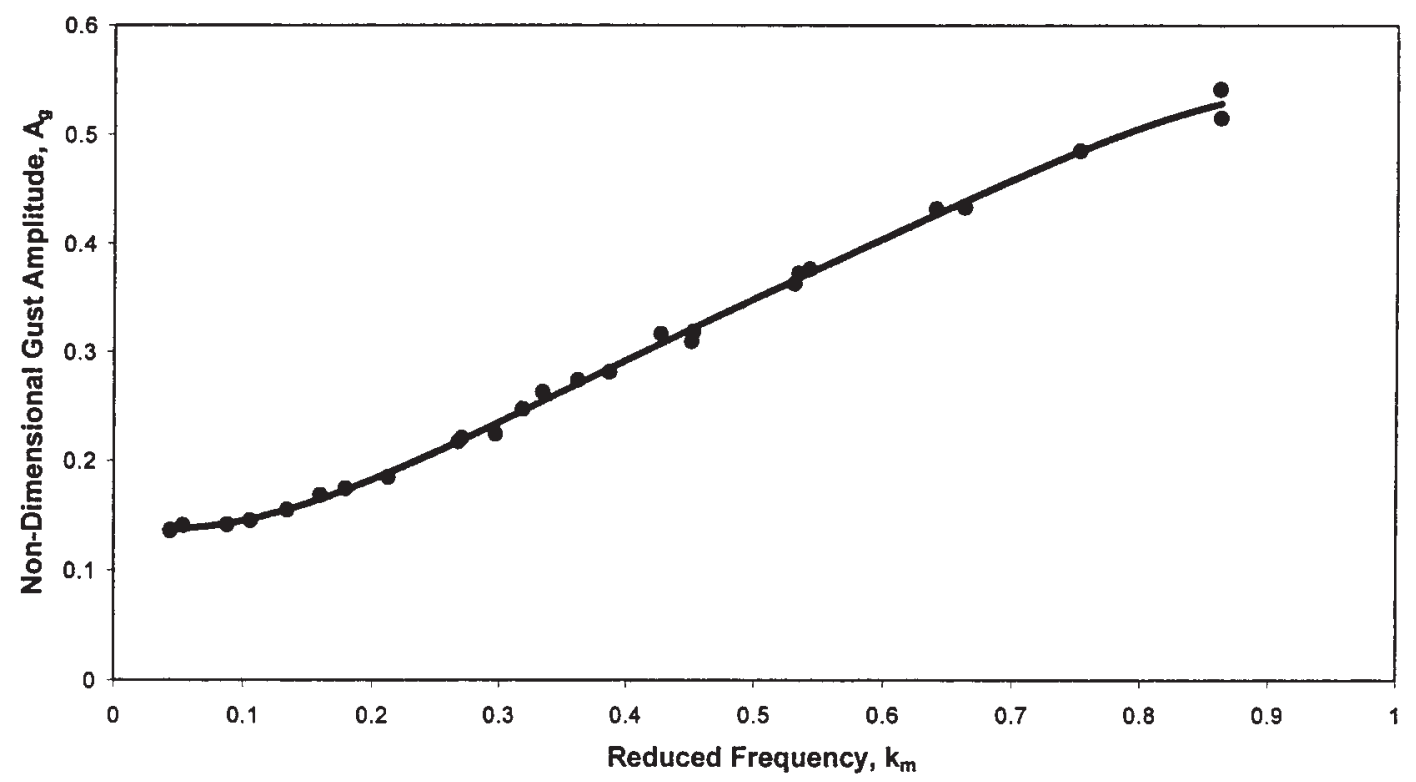

Fig. 2 Gust generator calibration

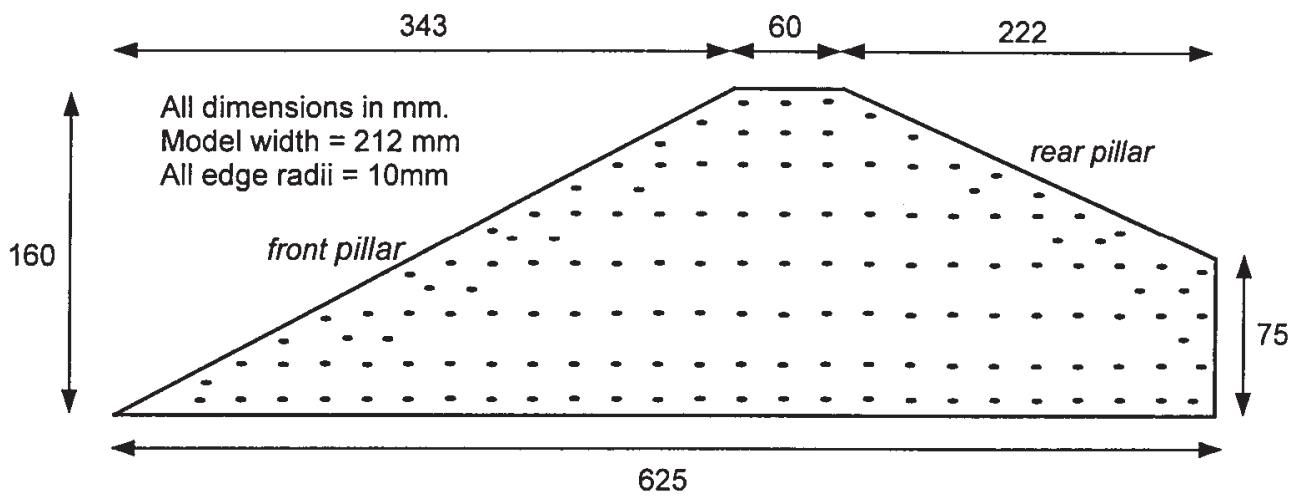

Fig. 3 Davis model dimensions and pressure tapping locations

full vehicle scale. The model is a simplified bluff body with a variable back-slant angle. The latter feature allows all of the primary flow features seen in automotive aerodynamics to be generated. However, the results reported here are limited to a single back angle of $20^{\circ}$. The model was constructed from a thin GRP shell and is mounted $40 \mathrm{~mm}$ from a ground board with the front of the model placed 1.3 model lengths downstream of the aerofoil trailing edges. The ground board extends 0.6 model lengths in front of the model and 2.4 model lengths downstream. The support sting enters through the model underside, thereby minimizing the aerodynamic interference. The blockage ratio and the Reynolds number based on length are 2.3 per cent and $1 \times 10^{6}$ respectively. One side of the model was fitted with pressure tappings by fitting brass tubing with an internal diameter of $0.8 \mathrm{~mm}$ ensuring that they were flush with the outer skin. A total of 144 tappings were arranged on a mainly $25 \mathrm{~mm}$ grid, but the density was increased along the front and rear pillars where the flow development is likely to be critical.
In order to allow comparison of the steady state data the model is further equipped with a six-component internal strain gauge balance. All data are acquired using a PC-based data acquisition system running a proprietary software package.

\section{STEADY STATE MEASUREMENTS}

Steady state measurements were conducted over the yaw angle range from $+10^{\circ}$ to $-10^{\circ}$ in steps of $2^{\circ}$ using a conventional differential pressure transducer connected via a rotary scanning valve. A wall static aligned with the model was used as the reference. As the model and tunnel are symmetrical, the readings for positive yaw can be used as the windward data and the negative as the leeward data.

For each tapping location $\Delta C_{p}$ values were determined by comparing the pressure coefficients on the windward and leeward sides. By assigning an area around each tapping such that the complete model side is accounted 


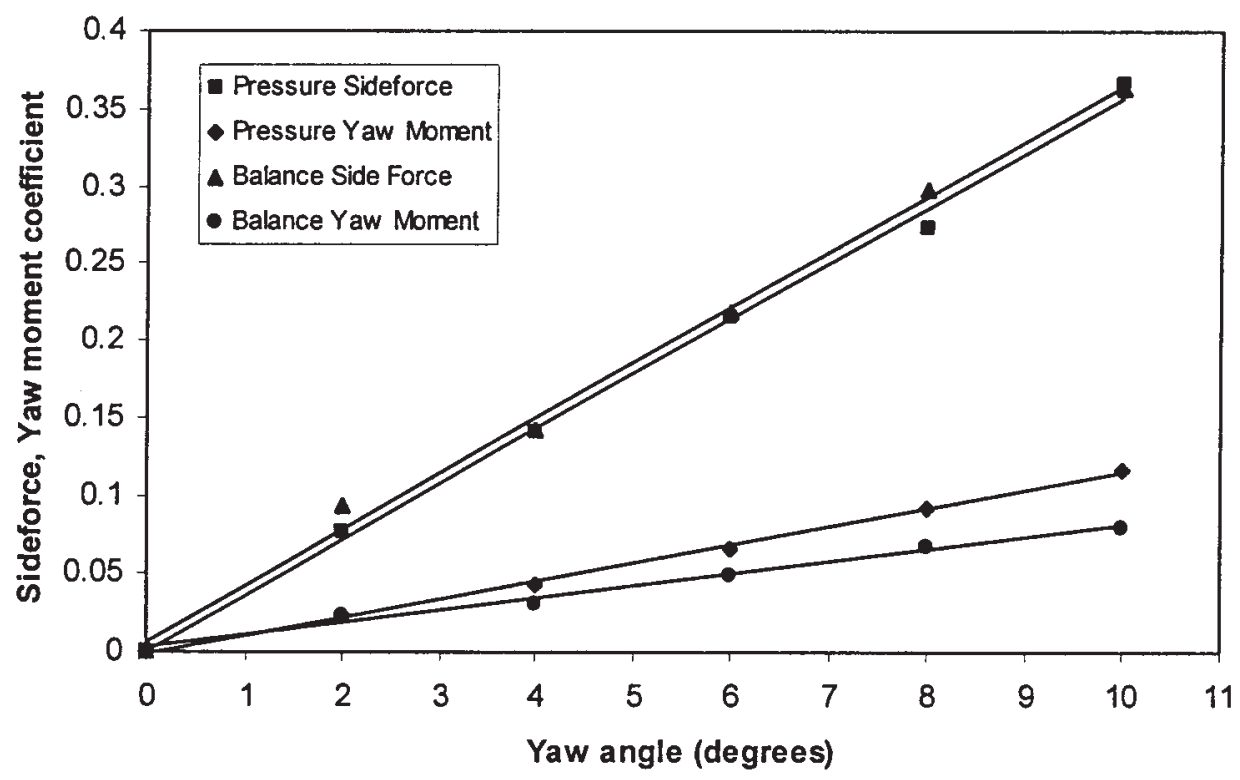

Fig. 4 Steady state results

for, the $\Delta C_{p}$ values can be integrated to obtain the total side force. Further inclusion of a moment arm relative to mid-model for each tapping allows the calculation of the yaw moment. The results are shown in Fig. 4 with balance-derived results included for comparison. The balance measurements include the skin friction contribution, which arises from cross-flow when the model is yawed. For a bluff body of this type the skin friction would generally be estimated at between 10 and 15 per cent of the total. Further discrepancies between the two methods may be introduced through the finite number and the positioning of the tappings, particularly along the edges of the model.

If it is assumed that the transient response can be inferred from the steady data (quasi-steady response) then the coefficient gradients from Fig. 4 may be interpreted as a measure of the cross-wind susceptibility of the model. The side force coefficient gradients $\mathrm{d} C_{y} / \mathrm{d} \beta$ and the yaw moment coefficient gradients $\mathrm{d} C_{\mathrm{Mz}} / \mathrm{d} \beta$ are summarized in Table 1.

For the purposes of determining the aerodynamic magnification factor $\left(\chi_{\mathrm{a}}\right)$ the values obtained from the pressure data are used to generate the quasi-steady response. This ensures a direct comparison with the pressure measurements made during the transient tests.

Contour plots of the surface pressure are shown in Fig. 5. As the yaw angle increases, the pressure is seen to increase over the windward side, apart from the area of the rear pillar, where the low pressure region associ-

Table 1 Steady state coefficient gradients

\begin{tabular}{lll}
\hline & $\mathrm{d} C_{y} / \mathrm{d} \beta$ & $\mathrm{d} C_{\mathrm{M} z} / \mathrm{d} \beta$ \\
\hline Pressure data & 0.0358 & 0.0115 \\
Balance data & 0.0358 & 0.0078 \\
\hline
\end{tabular}

D10600 (C) IMechE 2001 ated with the trailing vortex system is intensified. At the front pillar the weak vortex has dissipated by about $6^{\circ}$ of yaw. On the leeward side the rear pillar vortex is seen to decrease with increasing yaw angle while at the front pillar it deepens considerably.

These plots would indicate that the rapid increase in side force seen in Fig. 4 is dominated by changes at the front of the model, thereby also giving rise to the increase in yaw moment. This is confirmed in Fig. 6 where the front side force coefficient is seen to rise much more rapidly than the rear.

\section{TRANSIENT MEASUREMENTS}

The transient data were acquired using four small solid state differential pressure transducers located inside the model and connected via short lengths of tubing to the tapping and a longer tube to the wall static reference pressure. The transducer outputs were amplified via high gain amplifiers and filtered using $100 \mathrm{~Hz}$ low pass filters. The short lengths of tubing and relatively low frequencies being considered ensured that there was no requirement to apply a correction to the data as proposed by Sims-Williams [10]. The complete system for each transducer, amplifier and the data acquisition equipment was calibrated against the conventional pressure transducer used in the steady state measurements to ensure comparability. To acquire data for all 144 tappings the transducers were manually moved from one group of four tappings to the next four. To ensure time alignment of the measurements data acquisition was triggered by a pulse from the gust generator. Some on-line phaselocked averaging was performed and the averaged data were saved for subsequent analysis. Transient data were 


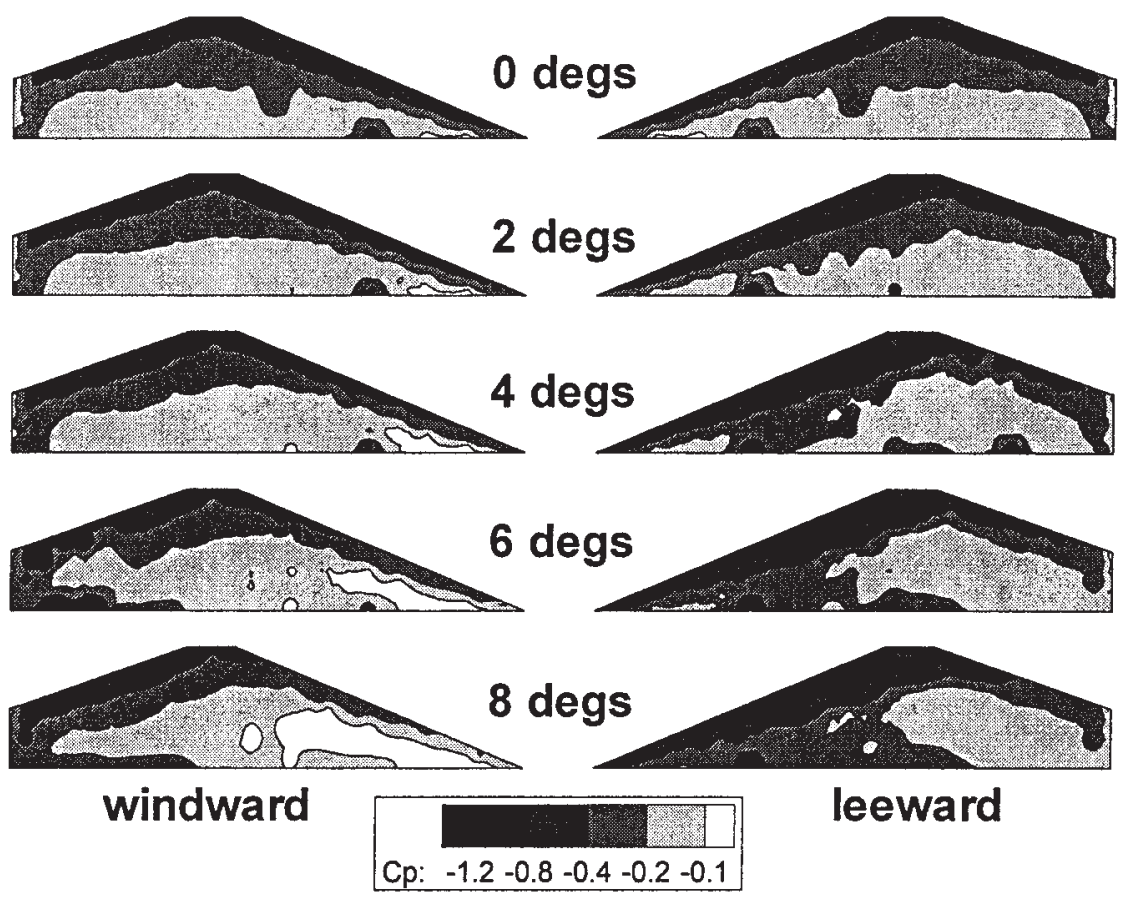

Fig. 5 Surface pressure contours for steady state yaw tests

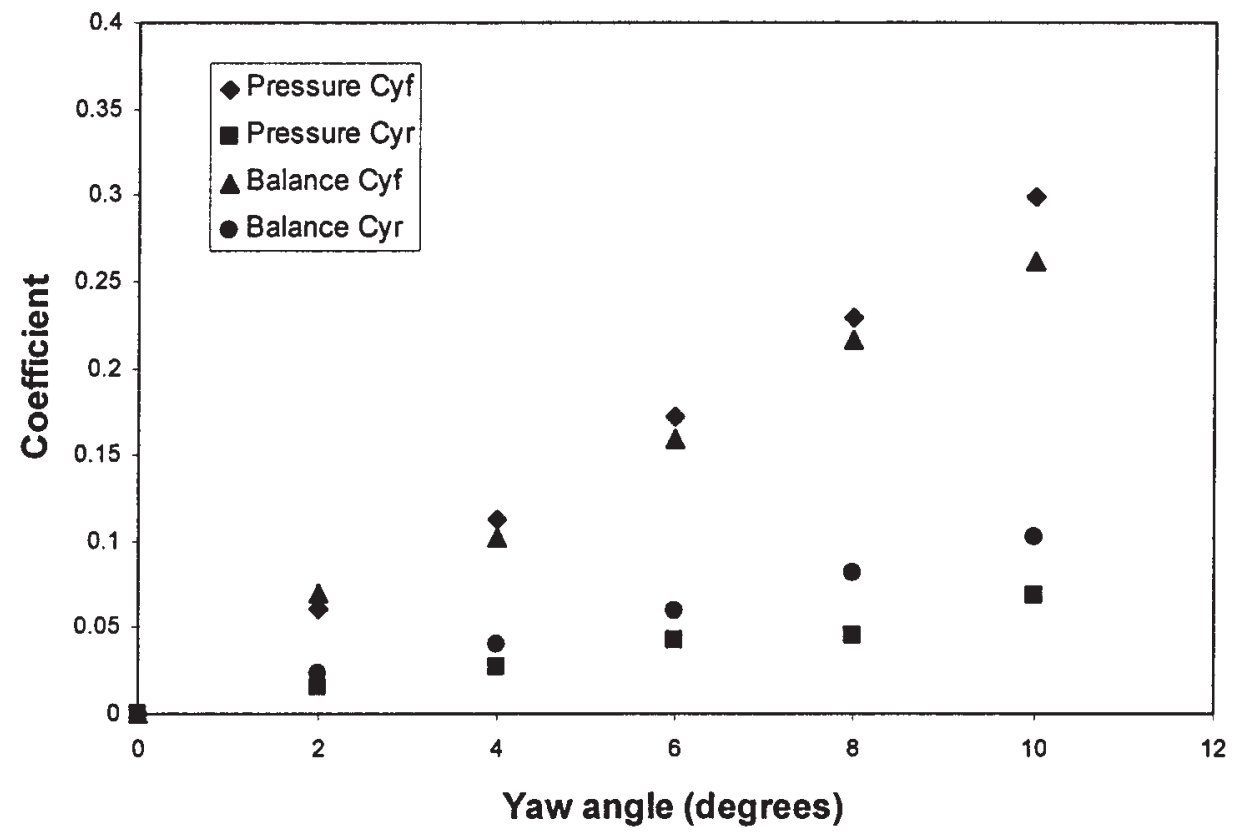

Fig. 6 Front and rear side force coefficients from steady state tests

acquired between 2 and $8 \mathrm{~Hz}$ corresponding to a reduced frequency range of $0.09-0.71$.

The data for a single tapping at a single frequency are converted to non-dimensional pressure coefficients $\left(C_{p}\right)$, and a second set representing the opposite side of the model is generated by applying a $180^{\circ}$ phase shift. Taking the difference produces a time series of $\Delta C_{p}$ for that location. An example plot is shown in Fig. 7.

The resulting data were found to have a sine wave form, in line with the findings of Mullarkey [8], who showed that the energy in the output signal was concentrated at the aerofoil frequency. On using this result the analysis is simplified by applying a sine fit to the data to determine amplitude, phase and frequency, where the frequency is equal to the aerofoil oscillation frequency. The large quantity of transient data is thereby reduced to simple sine parameters for each tapping. When the technique is applied to each tapping and the result is integrated, the transient side force and yaw moment are calculated, and this results in an overall sine response. 


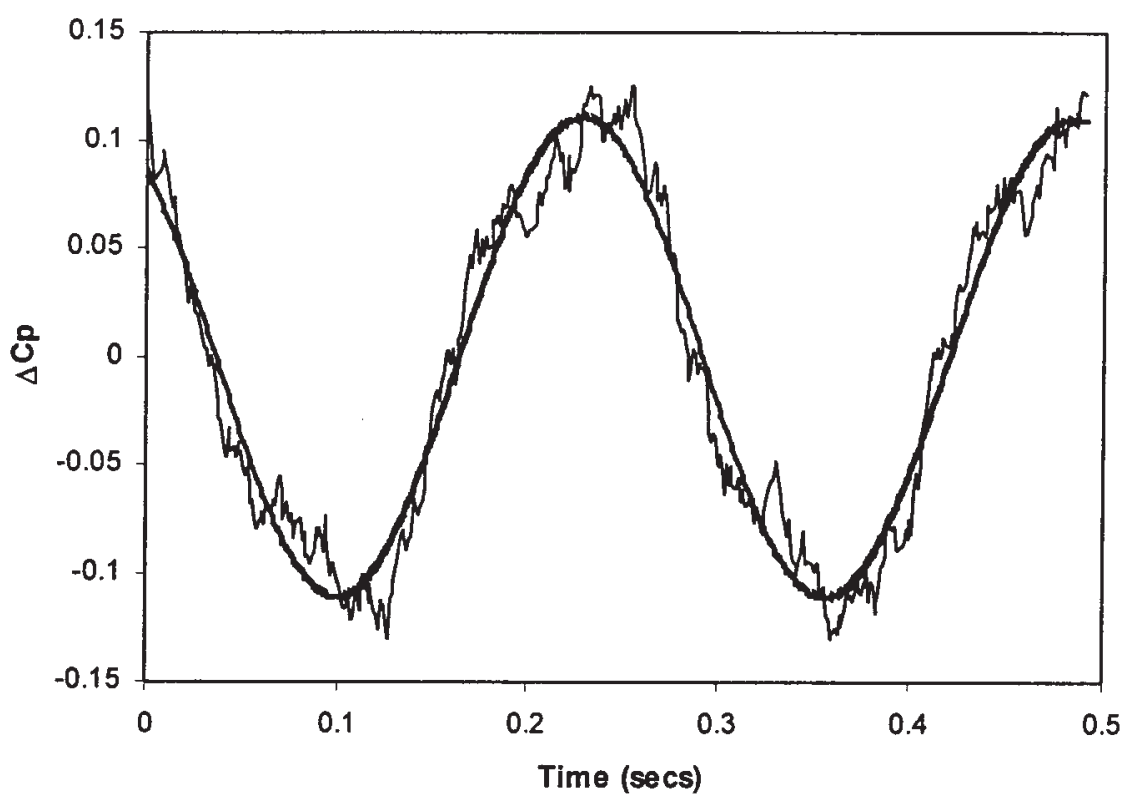

Fig. 7 Single-tapping transient $\Delta C_{p}$ variation and sine fit

With the output at the same frequency as the input the transient forces or moments can be compared directly with those predicted from a quasi-steady calculation. The requirement to determine signal power via the power spectral density is therefore removed.

A quasi-steady prediction can be generated by combining the known sinusoidal input and the steady state coefficient gradients. For the side force coefficient this is represented as

$$
C_{y}(t)=\frac{\mathrm{d} C_{y}}{\mathrm{~d} \beta} A_{\mathrm{g}} A_{0} \sin (\omega t)
$$

In practice, with the simplification in the analysis described above, the aerodynamic magnification factor $\left(\chi_{\mathrm{a}}\right)$ can be determined by simply taking the ratio of the amplitudes of the transient to the quasi-steady sine wave outputs. Aerodynamic admittance, if required, can be calculated by squaring this result.

The quasi-steady prediction calculated above assumes that the sinusoidal gust input acts to generate an equal cross-wind velocity and thereby yaw angle along the length of the model. This is a necessary assumption when the model response is determined from a force balance. However, with the use of pressure tappings it is possible to take account of the phase differences that occur along the length of the model and arise as the gust is convected at tunnel freestream speed. In this case, quasi-steady pressure data must be generated for each tapping using a steady state pressure coefficient gradient and the relative phase for each tapping. The latter is determined from the tapping longitudinal position and tunnel freestream speed:

$$
C_{p_{n}}(t)=\frac{\mathrm{d}\left(\Delta C_{p_{n}}\right)}{\mathrm{d} \beta} A_{\mathrm{g}} A_{0} \sin \left(\omega t+\psi_{n}\right)
$$

The individual tapping predictions are integrated to produce a predicted side force and yaw moment and combined with the transient result to determine $\chi_{\mathrm{a}}$. Figure 8 summarizes the aerodynamic magnification results for side force and yaw moment.

Across the majority of the frequency range, the quasisteady prediction overestimates the transient side force; the exception is at the lowest reduced frequency where the aerodynamic magnification rises to 1.5 . The yaw moment magnification exceeds 1 across the frequency range by between 5 and 30 per cent, suggesting some transient flow effects. The inclusion of the phase information has little effect on the side force but increases yaw moment $\chi_{\mathrm{a}}$ at higher frequencies. At the highest frequency tested, the wavelength of the gust is of the order of 5 model lengths, and the calculated phase difference between the front and back of the model is therefore of the order of $80^{\circ}$. The trend, seen in other work [3], of a reducing magnification factor at low reduced frequencies is not seen here; in fact, both the yaw moment $\chi_{\mathrm{a}}$ and side force $\chi_{\mathrm{a}}$ are well above unity at the lowest frequency tested. It is noted, however, that in common with other work of this type the nondimensional gust amplitude decreases with decreasing frequency. In the design used here at the lowest reduced frequency the gust amplitude is less than $\pm 2^{\circ}$, giving rise to some concern regarding the overall signal-to-noise ratio. Modifications to the gust generator in order to obtain a constant gust amplitude across a range of frequencies are a possibility for future work but would not resolve the problem of poor signal-to-noise ratio.

Some further insight into the results in Fig. 8 may be gained by plotting the phase relationship of the response at a particular tapping with respect to the model leading edge. This is derived when fitting the $\Delta C_{p}$ response for 


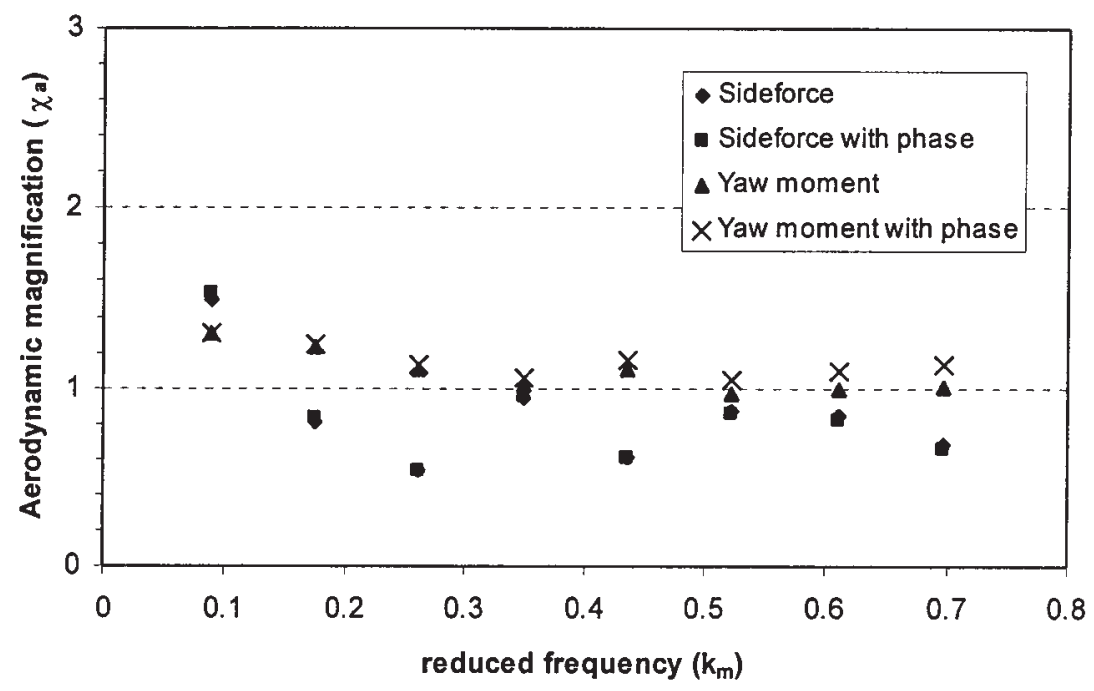

Fig. 8 Sideforce and yaw moment aerodynamic magnification against reduced frequency

each tapping. This is seen in Fig. 9; the phase effect of the gust convection has been removed.

As the operating frequency is reduced, increasingly large areas of the model side lag the quasi-steady response. Such a result is liable to give rise to an increase in yaw moment but may also result in a reduction in the side force, irrespective of the amplitude of the pressure variation at individual tappings. The existence of lag within the flow field is consistent with the highly viscous nature of the flow.

Time-aligned stills from an animation of the transient and quasi-steady pressure fields are shown in Fig. 10. These were acquired at the highest frequency tested. Although in many respects the two sets of plots are similar there is a noticeable increase in the intensity and extent of the three-dimensional features at the front and rear pillars in the transient case. This suggests not only that there is insufficient time for the flow to reach a steady condition but also that viscous action within the vortices serves to increase their strength.

Figure 11 shows contours of aerodynamic magnification generated by comparing the transient response at each tapping with the quasi-steady prediction. Care must be taken in the interpretation of these plots because the amplitude of the pressure fluctuation varies from point to point considerably. Equal magnification factors at two points may therefore not contribute equally to the overall effect. However, they are useful in identifying important areas of activity. At low frequencies, the magnification is highest at the rear of the model, with much of the front of the model showing magnification factors below 1. As the frequency is increased, the influence of the front becomes more important. Calculating front and rear side force magnification (from the side force and yaw moment data) confirms that at low frequencies the rear of the model dominates and at high frequencies the front dominates.

\section{CONCLUSIONS}

1. A series of experiments have been conducted to measure the steady and transient side force and yaw moment response of a simplified car-type bluff body. Sinusoidal approach flow was generated using an oscillating aerofoil gust generator, and the model response determined using surface pressure tappings.

2. Steady state coefficient gradients were determined

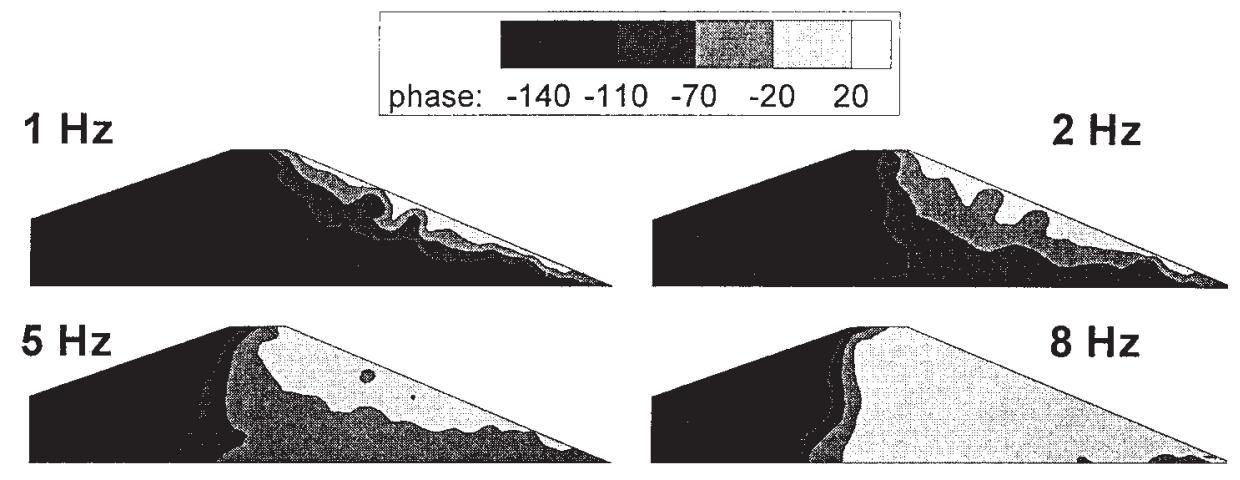

Fig. 9 Plot of phase relative to front of model 


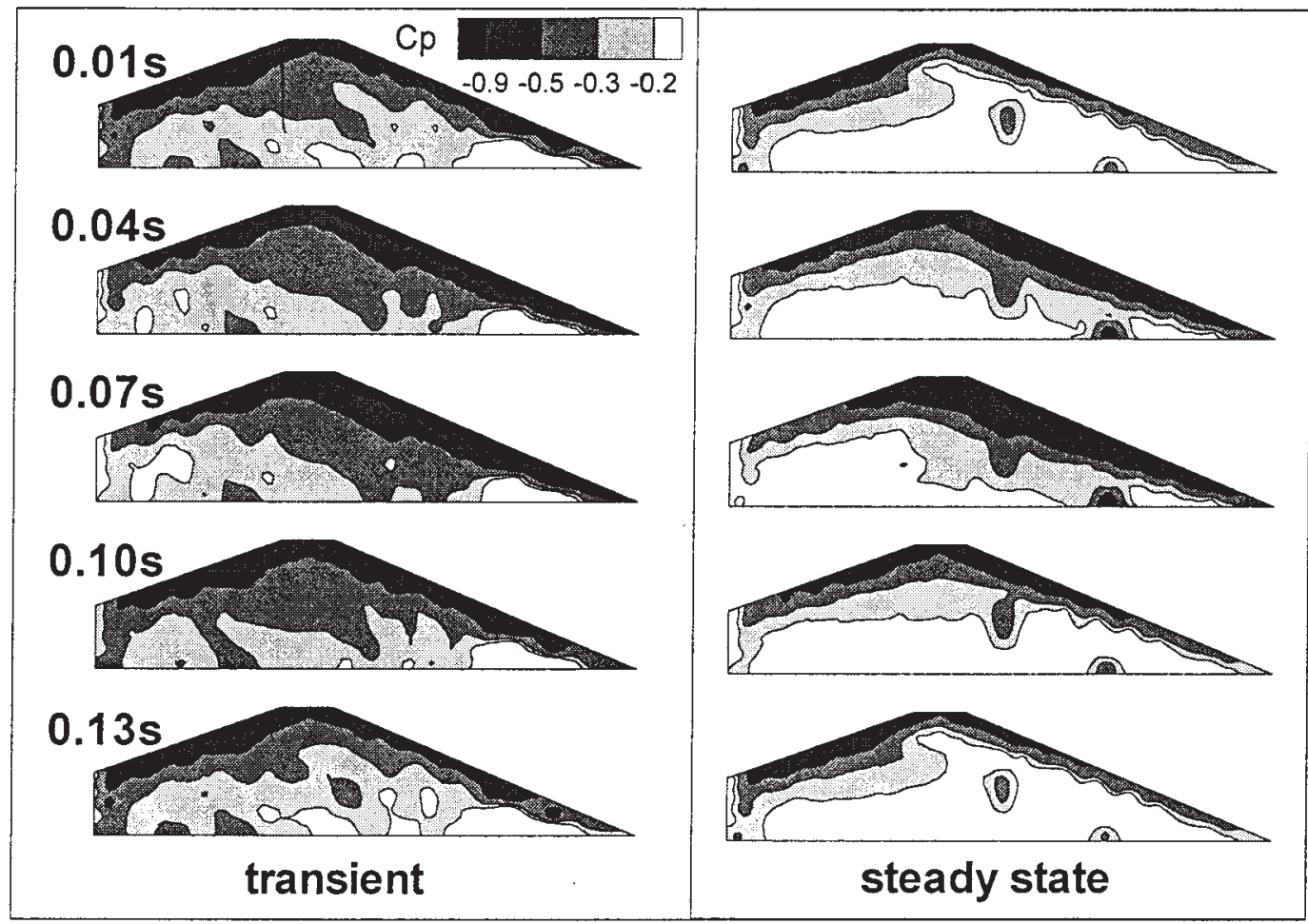

Fig. 10 Comparison of transient and quasi-steady pressure fields

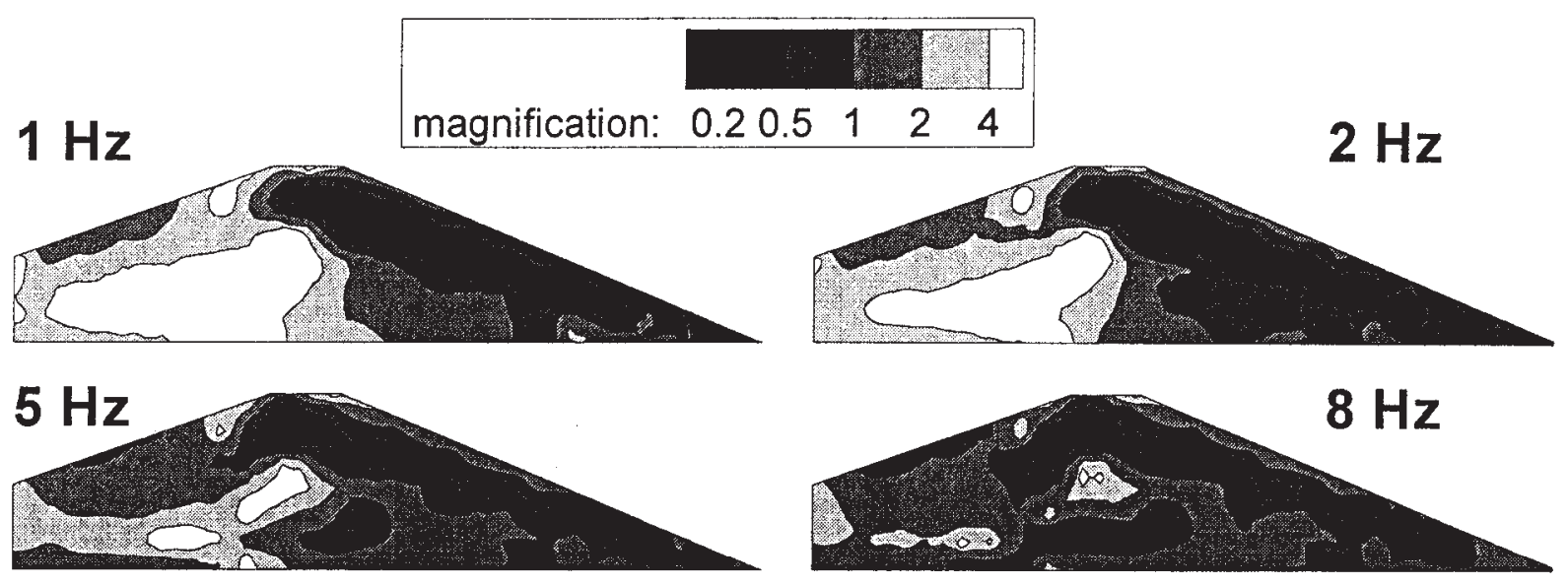

Fig. 11 Contours of aerodynamic magnification $\left(\chi_{\mathrm{a}}\right)$

in order to determine the quasi-steady response, and they compare well with those obtained using an internal strain-gauged balance.

3. Transient data acquired over a range of non-dimensional frequencies are analysed to determine side force and yaw moment aerodynamic magnification factors.

4. Yaw moment is underpredicted by quasi-steady prediction by between 5 and 30 per cent. Side force is overestimated by quasi-steady methods except at the lowest frequency tested.

5. It is concluded that the unsteady flow field does not reach the steady condition even at the lowest frequency tested where the gust wavelength is in excess of 35 model lengths. Viscous effects in regions such as the front and rear pillars serve to intensify the vortex structure and to modify the overall flow field.

6. Phase changes relative to the quasi-steady response further modify the transient response.

\section{ACKNOWLEDGEMENTS}

The authors would like to thank the technical staff in the Department of Aeronautical and Automotive Engineering for their work in the design and construction of the gust generator and model, in particular $\mathrm{Mr}$ K. Coulthard, Mr N. Randall, Mr P. Stinchcombe and 
Mr T. Newbold, and also for the encouragement from Mr A. Morby during the construction phase.

\section{REFERENCES}

1 Smith, N. P. Wind gusts measured on high speed roads. Proc. Instn Mech. Engrs, 1973, 187.

2 Watkins, S. and Saunders, J. W. Turbulence experienced by road vehicles under normal driving conditions. SAE paper 950997, Special Publication SP-1078, 1995.

3 Bearman, P. W. and Mullarkey, S. P. Aerodynamic forces on road vehicles due to steady side winds and gusts. In RaeS, Vehicle Aerodynamics Conference, Loughborough, 1994.

4 Macklin, A. R., Garry, K. P. and Howell, J. P. Comparing static and dynamic testing techniques for the crosswind sensitivity of road vehicles. SAE paper 960674, Special Publication SP-1145, 1996.
5 Garry, K. P. and Cooper, K. R. Comparison of quasi-static and dynamic wind tunnel measurements on simplified tractor-trailer models. J. Wind Engng Ind. Aerodyn., 1986, 22, 185-194.

6 Ryan, A. and Dominy, R. G. The aerodynamic forces induced on a passenger vehicle in response to a transient cross-wind wind gust at a relative incidence of $30^{\circ}$. SAE paper 980392, Special Publication SP-1318, 1998.

7 Davenport, A. G. The application of statistical concepts to the wind loading of structures. Proc. Instn Civil Engrs, 1961, 19, paper 6480, 449-471.

8 Mullarkey, S. P. Aerodynamic stability of road vehicles in side winds and gusts. PhD thesis, University of London, 1990.

9 Davis, J. P. Wind tunnel investigation of road vehicle wakes. PhD thesis, Imperial College of Science and Technology, University of London, October 1982.

10 Sims-Williams, D. B. and Dominy, R. G. Experimental investigation into unsteadiness and instability in passenger car aerodynamics. SAE paper 980391, 1998. 